PSICOLOGIA

IBEROAMERICANA
Psicología Iberoamericana ISSN: 1405-0943

revista.psicologia@ibero.mx

Universidad Iberoamericana, Ciudad de México México

\title{
Escala de bienestar psicológico para adultos mayores: Construcción y validación
}

de León Ricardi, César Augusto; García Méndez, Mirna; Rivera Aragón, Sofía

Escala de bienestar psicológico para adultos mayores: Construcción y validación

Psicología Iberoamericana, vol. 26, núm. 2, 2018

Universidad Iberoamericana, Ciudad de México, México

Disponible en: http://www.redalyc.org/articulo.oa?id=133959841003 


\title{
Escala de bienestar psicológico para adultos mayores: Construcción y validación
}

\author{
SCALE OF PSYCHOLOGICAL WELL-BEING FOR OLDER ADULTS: CONSTRUCTION AND \\ VALIDATION
}

César Augusto de León Ricardi ${ }^{1}$

Universidad Nacional Autónoma de México, México

Redalyc: http://www.redalyc.org/articulo.oa?

dricardi84@gmail.com

Mirna García Méndez

Universidad Nacional Autónoma de México, México

Sofía Rivera Aragón

Universidad Nacional Autónoma de México, México $\mathrm{id}=133959841003$

Recepción: 17 Diciembre 2018 Aprobación: 06 Marzo 2019

\section{Resumen:}

El bienestar psicológico es uno de los principales indicadores de la salud mental en diferentes etapas del ciclo vital, particularmente en la vejez. Tomando en cuenta la necesidad de contar con instrumentos válidos y confiables para medir constructos psicológicos, el objetivo de este estudio es desarrollar y validar una escala de bienestar psicológico para adultos mayores de la Ciudad de México. La muestra no probabilística intencional quedó conformada por 200 participantes, con un rango de edad de 60 a 90 años (Medad=67.9, DE=7.2). Como resultado del análisis factorial se obtuvieron 16 ítems distribuidos en cuatro factores que evalúan: control personal, seguridad personal, relaciones sociales y autonomía. La escala explica el 54.8\% de la varianza, con un alfa de Cronbach global de .71. La escala obtenida cumple con criterios psicométricos adecuados para medir el bienestar psicológico en adultos mayores de la Ciudad de México.

Palabras Clave: Bienestar psicológico, envejecimiento, escala, salud, gerontología.

\section{Abstract:}

Psychological well-being is one of the main indicators of mental health in different stages of the life cycle, particularly in old age. Considering the need for valid and reliable instruments to measure psychological constructs, the objective of this study is to develop and validate a scale of psychological well-being for adults over Mexico City. A probability sample of 200 participants between the ages of $60-90$ years (Mage $=67.9, \mathrm{SD}=7.2$ ) was used. Factorial analysis was used to analyze the data. Results showed 16 items distributed across four factors that evaluated personal control, personal security, social relations and autonomy. The scale explains $54.8 \%$ of variance, with an overall Cronbach alpha of .71. A scale was developed based on appropriate psychometric criteria to measure psychological well-being.

KEYWORDS: Psychological well-being, aging, scale, health, gerontology.

\section{INTRODUCCIÓN}

En México, para el año 2030 el número de adultos mayores de 60 años se duplicará y alcanzará los 20 millones (Gutiérrez, 2012). Este fenómeno de transición demográfica se ha denominado envejecimiento poblacional y se caracteriza por una continua disminución en las tasas de natalidad y de mortalidad, por lo que durante el periodo de transición demográfica la población de adultos mayores aumenta significativamente en comparación con la población infantil y juvenil. En años recientes, el envejecimiento poblacional se ha investigado con énfasis en la identificación de los factores y condiciones que promueven el envejecimiento

\section{NotAS DE AUTOR}

1 Universidad Nacional Autónoma de México, Av Universidad 3000, Cd. Universitaria, Coyoacán, 04510, Ciudad de México, cdmx. 
exitoso, entendiendo éste como el proceso en el que la salud física y mental continúan sin detrimento hasta la vejez (Fernández-Ballesteros, 2009). Desde una perspectiva psicológica, en la vejez el bienestar psicológico (bp) destaca por presentar asociaciones positivas con los siguientes indicadores de salud física y mental: autoestima (Hyun, Ju, \& Sohyune, 2012), satisfacción con la vida (Gutiérrez, Tomás, Galiana, Sancho, \& Cebria, 2013), la actividad física (Kahana, Kelley-Moorea, \& Kahana, 2012) y emociones positivas Huppert (2004).

El estudio del bienestar se ha organizado en dos tradiciones (Ryan \& Deci, 2001): la primera aborda la felicidad (bienestar subjetivo) y se denomina tradición hedónica; la segunda se relaciona con el desarrollo del potencial humano (bienestar psicológico) y se conoce como tradición eudaimónica. Los estudios sobre la relación de estas dos tradiciones indican que ambas poseen componentes individuales distintos, lo que sugiere que ambas son independientes (Chen, Jing, Hayes, \& Lee, 2013).

La eudaimónica se encarga del estudio del bp, tiene su origen en el concepto aristotélico de eudaimonia. Para Aristóteles, la búsqueda de la perfección, a través de la realización del verdadero potencial del ser humano, es el fin último de cada persona, relegando la búsqueda de la felicidad a un segundo término, ya que ésta se traduce en la satisfacción de los deseos personales, volviendo a los humanos esclavos o seguidores de sus deseos (Ryan \& Deci, 2001). De acuerdo con este punto de vista, cada individuo llega a la vida con talentos únicos, conocidos como daimon; la prueba central de la vida es reconocer y realizar esos talentos. Waterman (1993) señaló que, mientras la felicidad se define hedónicamente, la concepción eudaimónica de bienestar exhorta a la gente a vivir de acuerdo con su daimon o verdadero yo. En la tradición eudaimónica, el bp se centra en la autorrealización y se define en términos del grado en que una persona se encuentra en un funcionamiento psicológicamente pleno.

Ryff (1989) expuso uno de los elementos más importantes de la psicología positiva al desarrollar su modelo multidimensional de bp. Este modelo de bienestar eudaimónico yace en el supuesto de que los individuos se esfuerzan por funcionar plenamente y hacer realidad sus talentos únicos. Para desarrollarlo, utilizó tres teorías psicológicas clínicas de crecimiento personal, la madurez psicológica de Gordon Allport, el funcionamiento pleno de Carl Rogers y la autorrealización de Abraham Maslow (Díaz et al., 2006). Su modelo está compuesto por seis dimensiones con direcciones negativas o positivas: autoaceptación, relaciones positivas con otros, autonomía, dominio del ambiente, propositivo en la vida y crecimiento personal.

Cada dimensión del bp implica desafíos que las personas enfrentan con la finalidad de funcionar positivamente. Es decir, la gente trata de sentirse bien consigo mismos, incluso siendo conscientes de sus propias limitaciones (autoaceptación); también tratan de desarrollar y mantener relaciones interpersonales cálidas y de confianza (relaciones positivas con otros), se vinculan con su entorno con el fin de satisfacer sus necesidades y deseos personales (dominio del ambiente), además buscan un sentido de autodeterminación e independencia personal (autonomía), así como encontrar significado en los esfuerzos y desafíos propios (propósito en la vida) y aprovechar al máximo los talentos y capacidades (crecimiento personal).

El modelo de Ryff (1989) es tomado como el principal referente en los estudios del bp en diferentes disciplinas ya que ha demostrado tener un importante apoyo teórico y empírico.

Respecto a la relación entre el bp y el envejecimiento, algunas dimensiones muestran efectos de la edad. Diversos estudios como el realizado por Oliver, Navarro, Meléndez, Molina y Tomás (2009) con 1,397 adultos mayores dominicanos reportan que la edad afecta negativamente las dimensiones de autonomía y dominio del ambiente; así mismo Meléndez, Tomás y Navarro 2011) señalan que existe una correlación negativa entre la edad y las dimensiones de dominio del ambiente, crecimiento personal, relaciones positivas, autoaceptación y propósito vida. Respecto a las dimensiones del bp que permanecen relativamente estables con la edad, Triadó, Osuna, Resano, y Villar (2003) refieren que la autoaceptación y las relaciones con otros no presentan diferencias entre grupos de edades diferentes, mientras que las dimensiones de dominio del entorno y autonomía presentan un patrón similar de estabilidad a partir de la mediana edad a la vejez (Mayordomo, Sales, Satorres, \& Meléndez, 2016). 
En cuanto a la medición del bp, es importante señlar que se han desarrollado diversos instrumentos (ver tabla 1) con adecuadas propiedades psicométricas, los cuales permiten medir múltiples dimensiones del bienestar, como el material, personal o de pareja. De estos instrumentos, la escala de Ryff(1989) compuesta por seis dimensiones (autoaceptación, relaciones positivas con otros, autonomía, dominio del ambiente, propositivo en la vida y crecimiento personal) es ampliamente utilizada en investigaciones dentro del campo de las ciencias sociales y de la salud.

TABLA 1

Escalas utilizadas para medir bienestar psicológico

\begin{tabular}{|c|c|c|}
\hline Nombre & Medida & Características \\
\hline $\begin{array}{l}\text { Bienestar psicológico } \\
\quad \text { (Riff, 1989) }\end{array}$ & Bienestar psicológico & $\begin{array}{l}\text { Escala con altas correlaciones entre algunas } \\
\text { dimensiones en diferentes validaciones }(\alpha=.84)\end{array}$ \\
\hline $\begin{array}{l}\text { Escala de eudemon de bienestar } \\
\text { personal (Fierro \& Rando, 2007) }\end{array}$ & Bienestar personal & $\begin{array}{l}\text { Compuesta por las dimensiones ausencia de } \\
\text { bienestar personal, y existencia de bienestar positivo } \\
\qquad(\alpha=.94)\end{array}$ \\
\hline $\begin{array}{l}\text { Escala de bienestar psicológico } \\
\text { (Sánchez-Cánovas, 2013) }\end{array}$ & $\begin{array}{l}\text { Evaluación del bienestar } \\
\text { psicológico subjetivo }\end{array}$ & $\begin{array}{l}\text { Compuesta por las subescalas de bienestar material, } \\
\text { laboral y de pareja }(\alpha=.94)\end{array}$ \\
\hline $\begin{array}{l}\text { Escala de rutas de acceso al } \\
\text { bienestar (Castro, 2011) }\end{array}$ & $\begin{array}{l}\text { Evaluación del bienestar } \\
\text { eudaimónico y hedónico }\end{array}$ & $\begin{array}{l}\text { Autoinforme que evalúa vida placentera }(\alpha=.81) \text {, } \\
\text { vida comprometida }(\alpha=.82) \text { y vida con significado } \\
\qquad(\alpha=.71)\end{array}$ \\
\hline
\end{tabular}

Su escala se ha traducido y adaptado a diferentes idiomas como el español (Díaz et al., 2006), italiano (Ruini, Ottolini, Rafanelli, Ryff, \& Fava, 2003), portugués (Fernández, Vasconcelos-Raposo, \& Teixeira, 2010) entre otros. A pesar del amplio uso de la escala de Ryff (1989), actualmente existe una discusión en el número total de dimensiones y reactivos que la integran, debido a las altas correlaciones que se presentan, como dominio del entorno y crecimiento personal (Díaz et al., 2006; Dierendonck, 2005; Tomas, Meléndez, Oliver, Navarro, \& Zaragoza, 2010).

En México, los estudios sobre medición del bp se han realizado a través de diferentes validaciones y adaptaciones de la escala de Ryff (1989), particularmente con adolescentes y jóvenes. Loera-Malvaez, BalcázarNava, Trejo-González, Gurrola-Peña y Bonilla-Muñoz (2008) realizaron una validación en 208 adolescentes con edades de 14 a 18 años, sus resultados mostraron que las seis dimensiones originales de la escala se agruparon en cuatro dimensiones con adecuadas propiedades psicométricas. Por otra parte, Medina-Calvillo, Gutiérrez-Hernández y Padrós-Blázquez, (2013) realizaron un estudio de las propiedades psicométricas de la escala de Ryff (1989) en dos muestras de jóvenes con una medida de edad de 19 y 29.46 años. Los resultados revelaron que en población mexicana el modelo teórico de bp de Ryff(1989) no presenta un ajuste adecuado, resultados similares a los reportados por Valenzuela (2015) quien evaluó la estructura factorial de la escala en jóvenes mexicanos.

Los instrumentos descritos tienen adecuadas propiedades psicométricas, pero fueron diseñados en otras culturas, demandan conocimiento numérico, adecuada comprensión del formato de repuesta y se han moldeado para poblaciones que difieren ampliamente de las características sociodemográficas de la población adulta mayor en México. Por tal motivo y aunado al creciente número de personas de la tercera edad y la falta de instrumentos que midan bp en adultos mayores en el contexto mexicano, con la intención de contribuir al desarrollo de escalas que evalúen constructos psicológicos en la vejez, el objetivo del presente estudio fue desarrollar y validar una escala de bp para adultos mayores de la Ciudad de México, el sustento teórico en el que se fundamenta este estudio fue el modelo mutidimensional de bp de Ryff (1989). 


\section{MÉTODO}

\section{Participantes}

Colaboraron de manera voluntaria 200 adultos mayores residentes de la Ciudad de México, con edades de 60 a 90 años $\left(M_{\text {edad }}=67.9, D E=7.2\right)$, del total de la muestra $51.5 \%$ eran hombres. Su nivel de escolaridad fue $68 \%$ con educación básica, $10.5 \%$ con educación media y $21.5 \%$ con educación superior. En relación con el estado civil, $14 \%$ eran solteros, $53 \%$ casados, $5 \%$ divorciados y $28 \%$ viudos. Se consideró como criterio de inclusión tener 60 años o más, contar con educación básica y no estar institucionalizado. Los participantes se seleccionaron por un muestreo no probabilístico intencional.

\section{Instrumento}

Para la construcción del instrumento se realizó un estudio exploratorio por medio de entrevistas abiertas con 10 adultos mayores, con las cuales se identificó el universo de conductas y elementos significativos del constructo de bp en adultos mayores. Las preguntas se elaboraron a partir de las dimensiones del modelo propuesto de bienestar de Ryff(1989). Para el análisis de la información recabada, se resumieron e integraron las descripciones obtenidas; se analizó la frecuencia y pertinencia de las palabras dentro de las diferentes dimensiones del modelo de Ryff (1989).

Con los resultados obtenidos se creó una base de 40 reactivos, resultado del acuerdo entre investigadores en psicología expertos en psicometría. Todos los reactivos contaron con un formato de respuesta tipo Likert pictórico, en el que se utilizaron figuras geométricas de diferentes tamaños como opciones de respuesta, ordenadas de mayor a menor y de izquierda a derecha. La calificación asignada a la escala fue de 1 a 4 puntos, se asignó 1 al cuadro más pequeño que implicaba menor cantidad del atributo (totalmente en desacuerdo) y 4 al cuadro más grande que implicaba mayor cantidad del atributo (totalmente de acuerdo); los reactivos se colocaron en orden aleatorio.

\section{Procedimiento}

Se solicitó la colaboración voluntaria de los participantes en centros de trabajo, hogares, parques y plazas públicas. En todos los casos se señaló que la información proporcionada era confidencial y anónima por lo que se omitieron los nombres de los participantes. A quienes accedieron a participar, se les entregó el instrumento y se solicitó que firmaran el consentimiento informado. El evaluador estuvo presente durante la aplicación del instrumento para resolver las dudas que pudieran surgir en el proceso, el cual se realizó de forma individual. Una vez terminada la aplicación de la escala, se agradeció a los participantes.

\section{RESULTADOS}

Para conocer la discriminación de los reactivos de la escala se siguió el proceso de validación psicométrica sugerido por Reyes-Lagunes, García y Barragán (2008). Para cada uno de los reactivos se realizó un análisis de frecuencias, sesgo y discriminación de reactivos para grupos extremos mediante una prueba t de Student, de los 40 reactivos sólo 16 cumplieron con las especificaciones requeridas para los análisis posteriores.

Se calculó la correlación reactivo-escala total, los resultados fueron de .29 a .53 . Debido a estas puntuaciones obtenidas no se eliminó ningún reactivo y se procedió a realizar un análisis factorial 
exploratorio de componentes principales con rotación ortogonal (varimax) para conocer el porcentaje de varianza explicada de la escala y el número de factores que la componen.

Los resultados obtenidos señalan que la medida de la adecuación del tamaño de muestra Kaiser-MeyerOlkin fue adecuada $(k m o=.759)$, mientras que la prueba de esfericidad de Bartlett's fue significativa $\left(\mathrm{X}_{2}=762,547, \mathrm{gl}=120, \mathrm{p}<.000\right)$ por lo que la escala es factorizable. Los 16 reactivos se agruparon en cuatro factores con pesos factoriales igual o superiores a 0.40 (ver tabla 2), el primer factor contiene los ítems 15,11, 9, 14 y 24, el segundo factor integra los ítems 12, 2, 22 y 7, el tercer factor contiene los ítems 4, 5, 26, 3 y el cuarto factor está compuesto por los ítems 17, 21 y 18. En conjunto explican el 54.80\% de la varianza total. Posteriormente, se realizaron análisis de confiabilidad mediante el coeficiente alfa de Cronbach, para obtener la consistencia interna global de la escala y la consistencia por factor. Se obtuvo una puntuación global de $\alpha=.77$. Los pesos factoriales y alfas por factor se presentan en la tabla 2 .

TABLA 2

Análisis factorial de la escala de bienestar psicológico para adultos mayores

\begin{tabular}{lcccc}
\hline \multicolumn{1}{c}{ Reactivos } & \multicolumn{3}{c}{ Factores } \\
\cline { 2 - 5 } & 1 & 2 & 3 & 4 \\
\hline 15. Considero que con los años he crecido como persona. & .753 & .076 & .011 & .091 \\
\hline 11. Puedo controlar los problemas que se me presentan. & .747 & .266 & -.048 & .064 \\
\hline 9. Siempre cumplo mis objetivos personales. & .641 & .246 & .077 & -.074 \\
\hline 14. Me siento bien con mi cuerpo. & .629 & .192 & .184 & .348 \\
\hline 24. Actualmente puedo trabajar. & .451 & .000 & .172 & .399 \\
\hline 12. Siempre estoy seguro(a) de mis acciones. & .309 & .782 & .077 & .047 \\
\hline 2. Tengo confianza para expresar lo que pienso. & -.011 & .723 & .159 & .207 \\
\hline 22. A pesar de los cambios, me gustan mis atributos físicos. & .253 & .633 & .072 & .318 \\
\hline 7. Soy una persona segura de sí misma. & .296 & .605 & .093 & -.118 \\
\hline 4. En comparación con otras personas tengo muchos amigos. & .150 & -.091 & .769 & .087 \\
\hline 5. Mis amistades son confiables. & -.032 & .195 & .733 & .039 \\
\hline 26. Me gusta conocer gente nueva. & .080 & .045 & .725 & .051 \\
\hline 3. Mis amistades son duraderas. & .016 & .219 & .690 & -.134 \\
\hline 17. Soy una persona autosuficiente. & -.022 & .287 & .016 & .717 \\
\hline 21. Soy libre para tomar mis propias decisiones. & .009 & .077 & -.057 & .699 \\
\hline 18. Soy independiente económicamente. & .220 & -.046 & .031 & .552 \\
\hline Varianza explicada & 15.38 & 14.13 & 14.07 & 11.21 \\
\hline Varianza acumulada & 15.38 & 29.51 & 43.59 & 54.80 \\
\hline Alfa de Cronbach & .71 & .73 & .71 & .60 \\
\hline Media & 3.01 & 3.15 & 2.91 & 3.02 \\
\hline Desviación estándar & 0.59 & 0.65 & 0.74 & 0.69 \\
\hline
\end{tabular}

Los cuatro factores obtenidos en el análisis factorial exploratorio se definieron de la siguiente forma, control personal (factor 1): grado de dominio personal y ambiental que posee un individuo; seguridad personal (factor 2): grado de seguridad física y mental que tiene un individuo; relaciones sociales (factor 3): grado en el que una persona logra mantener vínculos sociales confiables y duraderos; finalmente autonomía (factor 4): grado de independencia personal y económica que posee un individuo. Los reactivos asociados a domino del entorno se agruparon en el factor denominado control personal, mientras que el factor propósito en la vida no forma parte de la escala.

A partir de los resultados obtenidos en el análisis factorial exploratorio se realizó un análisis factorial confirmatorio mediante el programa de ecuaciones estructurales amos (versión 19) en el que se utilizó 
el método de máxima verosimilitud. Después de realizar los ajustes pertinentes que consistieron en la eliminación de los reactivos: 2. Tengo confianza para expresar lo que pienso, 24. Actualmente puedo trabajar y 9. Siempre cumplo mis objetivos personales, se obtuvo el modelo que se muestra en la figura 1 . Los índices de bondad de ajuste obtenidos fueron: $\mathrm{X}_{2} / \mathrm{gl}=1.21 ; \mathrm{NFI}=.873$; $\mathrm{IFI}=.975 ; \mathrm{CFI}=.974$, rmsea $=.033$ y srmr $=.06$. Estos valores indican ajustes adecuados del modelo (Hu \& Bentler, 1999).

Por último, con la intención de identificar si la escala puede detectar diferencias entre grupos, se realizó una prueba $t$ de Student para grupos independientes entre hombres y mujeres. Los resultados señalan que existen diferencias estadísticamente significativas en seguridad personal, $t(198)=3.207, \mathrm{p}<0.05$ y en relaciones sociales $\mathrm{t}(198)=-2.230, \mathrm{p}<.05$. Son las mujeres $(\mathrm{M}=3.30, \mathrm{DE}=.63)$ quienes muestran mayor grado de seguridad física y mental que los hombres $(\mathrm{M}=3.01, \mathrm{DE}=.64)$, mientras que los hombres $(\mathrm{M}=3.12$, $\mathrm{DE}=.67)$ presentan mayores vínculos sociales confiables y duraderos que las mujeres $(\mathrm{M}=2.90, \mathrm{DE}=.69)$. No se obtuvieron diferencias estadísticamente significativas en autonomía $(\mathfrak{t}(198)=-.39$, $\mathrm{p}>.05)$ y control personal $(\mathfrak{t}(198)=.33, \mathrm{p}>.05)$.

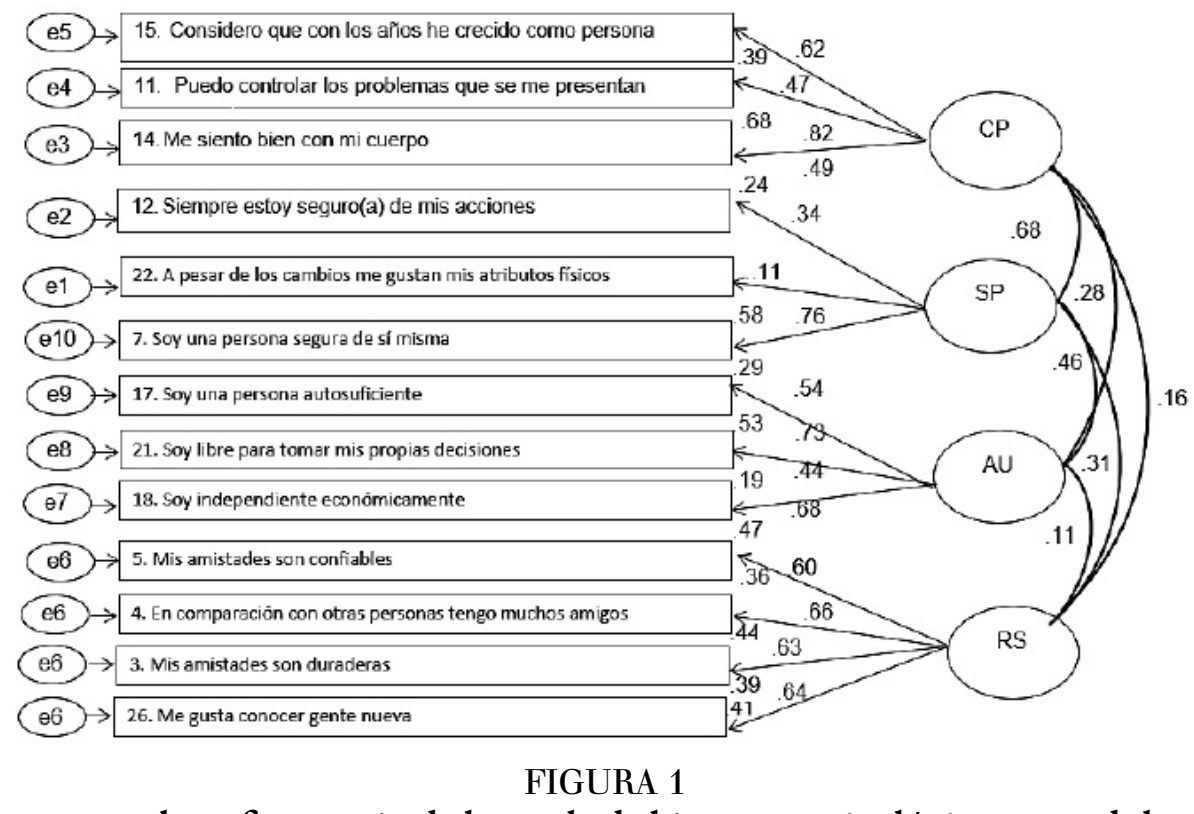

Modelo estructural confirmatorio de la escala de bienestar psicológico para adultos mayores. $\mathrm{cp}=$ control personal, $\mathrm{sp}=$ seguridad personal, $\mathrm{au}=\mathrm{autonomía}, \mathrm{rs}=$ relaciones sociales

\section{DISCUSIÓN}

La presente investigación tuvo como objetivo construir un instrumento de medición de cp para adultos mayores de la Ciudad de México. Respecto a la estructura factorial obtenida, los resultados sugieren que la escala se constituye de cuatro factores, los cuales mostraron correlaciones positivas, moderadas y bajas entre sí, lo que indica una probable independencia entre los factores que integran el constructo de cp en dicho sector de la población. Estos resultados coinciden con estudios previos realizados en México en los cuales se ha observado que el cp no se ajusta a la propuesta teórica de Ryff (1989) de seis factores; por ejemplo, en el análisis factorial confirmatorio realizado por Valenzuela (2015) el modelo que mostró mejores índices de ajuste fue el modelo integrado por dos factores, crecimiento personal y autoaceptación, mientras que en el estudio realizado por Loera-Malvaez et al. (2008) la estructura factorial del cp se conformó con cuatro componentes: autoaceptación, relaciones interpersonales, autonomía y satisfacción con la vida. Es importante señalar que estos estudios se han realizado principalmente en población adolescente y joven. Valenzuela (2015) refiere que este efecto de reducción de factores puede beberse al traslape en el modelo 
original de cuatro dimensiones (crecimiento personal, propósito en la vida, autoaceptación y dominio del entorno) en una sola, o posiblemente a la generación de sesgos en la estimación, producidos por el empleo de escalas ordinales y el uso de procedimientos analíticos que utilizan medidas continuas.

Por otra parte, los resultados de este trabajo destacan que en adultos mayores mexicanos el constructo cp no integra el factor dominio del entorno ni propósito en la vida, en relación con este hallazgo. Triadó et al. (2003) refieren que los cambios en estas dimensiones podrían deberse a que estos aspectos reciben un significado distinto conforme aumenta la edad, teniendo como resultado menor importancia en la experiencia personal. Además, estos resultados no coinciden con los estudios realizados en otros países (Fernández et al., 2010; Rivera, Quintero-Jiménez, Veray-Alicea, \& Rosario-Rodríguez, 2016) y estratos socioeconómicos (Ryff, 1989), lo que indica la importancia de considerar posibles efectos del contexto sociocultural y económico en futuros estudios sobre el cp en adultos mayores, ya que estas condiciones son un elemento central en el estudio del bienestar psicológico (Ryff, 1989).

La escala obtenida integra cuatro factores. El factor control personal agrupó reactivos asociados a la capacidad de desarrollo personal, control de situaciones y percepción positiva de las capacidades físicas. Estos reactivos se agrupan en lo que Waterman (1993) denominó como sentimientos de expresividad emocional, los cuales se relacionan con experiencias de compromiso las actividades de las personas, condiciones que crean una sensación plena de sentirse vivo y realizado.

Esta premisa refuerza la afirmación de que el desarrollo continuo, el crecimiento personal y estar abierto a nuevas experiencias constituyen aspectos importantes del funcionamiento psicológico óptimo. El factor de seguridad personal agrupó reactivos relacionados con la seguridad en las acciones, pensamientos y atributos físicos, condiciones que, en conjunto, permiten una valoración positiva o negativa de cada individuo. En la vejez, estudios como el de Zavala, Vidal, Castro, Quiroga y Klassen (2006) han demostrado que las personas que poseen una valoración positiva de sí mismos muestran un mejor ajuste en su funcionamiento social y familiar. En el factor de relaciones sociales se agruparon reactivos asociados a los lazos de amistad, afecto y confianza. En los adultos mayores, las relaciones sociales son importantes para proporcionar funciones de socialización, satisfacción de necesidades psicológicas básicas como el apego y sensación de una vida con significado (Charles \& Carstensen, 2009). Luo y Waite (2011) señalan que mientras más altos sean los niveles de apoyo, participación y sentimientos de conexión social, menores serán los niveles de estrés psicológico, mientras que en ausencia de relaciones sociales es común encontrar frecuentes síntomas depresivos (Charles \& Carstensen, 2009). En el factor de la autonomía se agruparon reactivos asociados a la valoración de independencia física, solvencia económica y la capacidad de tomar decisiones de forma autónoma, Lehr (1989) sugiere en su teoría de la vinculación que sólo se es feliz y se logra el bienestar cuando el adulto mayor se mantiene activo, es decir, que es capaz de realizar actividades de forma autónoma.

Respecto a los valores de consistencia interna, se obtuvieron resultados aceptables de alfa de Cronbach tanto para la escala total como para los factores que la integran con excepción del factor autonomía $(\alpha=.60)$. Este hallazgo coincide con los resultados de Medina-Calvillo et al. (2013) quien al validar la escala de bienestar de Ryff (1989) en población adolescente reportó bajos valores de consistencia interna en el factor de autonomía.

En relación con el efecto del sexo en el bp, en este estudio se encontró que las mujeres presentan mayores niveles de seguridad personal y los hombres mayores niveles de relaciones sociales, Rosa-Rodríguez, Negrón, Maldonado, Toledo y Quiñones (2015) refieren que estas diferencias pueden explicarse por las expectativas de rol de género asignadas a hombres y mujeres, por ejemplo, los hombres presentan mayor puntaje en dimensiones relacionadas con el contacto social y búsqueda de recursos externos, valores apreciados culturalmente en los hombres.

Finalmente, se sugiere que en futuras investigaciones se describa si la escala obtenida muestra correlaciones significativas con otras medidas de bienestar como autoestima, salud mental y bienestar subjetivo, igualmente se recomienda conocer si la escala permite identificar diferencias entre adultos mayores con y $\sin$ 
enfermedades mentales, es decir, realizar trabajos de validación en población clínica, dada la alta prevalencia de trastornos mentales en la vejez. Se concluye que la escala posee adecuados criterios psicométricos para medir bp en adultos mayores de la Ciudad de México.

\section{REFERENCIAS}

Castro, A. (2011). Las rutas de acceso al bienestar. Relaciones entre bienestar hedónico y eudaemónico. Un estudio en población argentina. Revista Iberoamericana de Diagnóstico y Evaluación Psicológica, 31(1), 37-57.

Charles, S., \& Carstensen, L. (2009). Social and emotional aging. Annual Review of Psychology, 61, 383-409. doi:10.1146/annurev.psych.093008.100448

Chen, F., Jing, Y., Hayes, A., \& Lee, J. (2013). Two concepts or two approaches? A bifactor analysis of psychological and subjective well-being. Journal of Happiness Studies, 14, 1033-1068. doi:10.1007/s10902-012-9367-x

Díaz, D., Rodríguez-Carvajal, R., Blanco, A., Moreno-Jiménez, B., Gallardo, I., Valle, C... Dierendonck, D. (2006). Adaptación española de las escalas de bienestar psicológico de Ryff. Psicothema, 18(3), 572-577.

Dierendonck, D. (2005). The construct validity of Ryff's scales of psychological well-being and its extension with spiritual well-being. Personality and Individual Differences, 36(2), 629-643. doi:10.1016/ S0191-8869(03)00122-3

Férnandez-Ballesteros, R. (2009). Gerontología social. Madrid: Piramide.

Fernández, H. Vasconcelos-Raposo, J., \& Teixeira, C. (2010). Preliminary analysis of the psychometric properties of Ryff s scales of psychological well-being in Portuguese adolescents. The Spanish Journal of Psychology, 13(2), 1032-1043. doi: $10.1017 /$ S1138741600002675

Fierro, A., \& Rando, B. (2007). Escala Eudemon de bienestar personal: Psicométricas. Anuario de Psicología, 38(3), 401-412.

Gutiérrez, L. (2012). La Academia Nacional de Medicina, el envejecimiento y la salud de los mexicanos. En L. Robledo \& D. Kershenobich. (Eds.), Envejecimiento y salud: Una propuesta para un plan de acción 2012 (pp. 17-25) México: Instituto Nacional de Geriatría.

Gutierrez, M., Tomás, J., Galiana, L., Sancho, P., \& Cebria, M. (2013). Predicting life satisfaction of the Angolan elderly: A structural model. Aging \& Mental Health, 17(1), 94-101. doi:10.1080/13607863.2012.702731

$\mathrm{Hu}, \mathrm{L} ., \quad \&$ Bentler, P. (1999). Cutoff criteria for fit indexes in covariance structure analysis: Conventional criteria versus new alternatives. Structural Equation Modeling: A Multidisciplinary Journal, 6(1), 1-55. doi:10.1080/10705519909540118

Huppert, A. (2004). Well-being: Towards an integration of psychology, neurobiology and social science. Philosophical Transactions of the Royal Society of London, 359, 1447-1451. doi:10.1098/rstb.2004.1520

Hyun, C., Ju, S., \& Sohyune, S. (2012). Factors influencing the successful aging of older Korean adults. Contemporary Nurse, 41(1), 78-87. doi:10.5172/conu.2012.41.1.78

Kahana, E., Kelley-Moorea, \& Kahana, B. (2012). Proactive aging: A longitudinal study of stress, resources, agency, and well-being in late life. Aging \& Mental Health, 16(4), 438-451. doi:10.1080/13607863.2011.644519

Lehr, U. (1988). Psicología de la Senectud. Barcelona: Herder.

Loera-Malvaez, N., Balcázar-Nava, P., Trejo-González, L., Gurrola-Peña, G., \& Bonilla-Muñoz, M. (2008). Adaptación de la escala de bienestar psicológico de Ryff en adolescentes preuniversitarios. Neurología, Neurocirugía y Psiquiatría, 41(3-4), 90-97.

Luo, Y., \& Waite, L. (2011). Mistreatment and psychological well-being among older adults: Exploring the role of psychosocial resources and deficits. Journal of Gerontology Series B, 66(2), 217-29. doi:10.1093/geronb/gbq096

Mayordomo, T., Sales, A., Satorres, E., Meléndez, J. (2016). Bienestar psicológico en función de la etapa de vida, el sexo y su interacción. Pensamiento Psicológico, 14(2), 101-112.

Medina-Calvillo, M., Gutiérrez-Hernández, C., \& Padrós-Blázquez, F. (2013). Propiedades psicométricas de la escala de bienestar psicológico de Ryff en población mexicana. Revista de Educación y Desarrollo, 27, 25-30. 
Meléndez, J., Tomás, J., \& Navarro, E. (2011). Actividades de la vida diaria y bienestar y su relación con la edad y el género en la vejez. Anales de Psicologia, 27(1), 164-169.

Oliver, A., Navarro, E., Meléndez, J., Molina, C., \& Tomás, J. (2009). Modelo de ecuaciones estructurales para predecir el bienestar y la dependencia funcional en adultos mayores de la República Dominicana. Revista Panamericana de Salud Publica, 26(3), 189-196.

Reyes-Lagunes, I. L., \& García y Barragán, L. F. (2008). Procedimiento de validación psicométrica culturalmente relevante: Un ejemplo. En S. Rivera, R. Díaz-Loving, R. Sánchez \& I. Reyes-Lagunes (Eds.), La psicología social en México, xii (pp. 625- 636). México: AMEPSO.

Rivera, J. A. G., Quintero-Jiménez, N., Veray-Alicea, J., \& Rosario-Rodríguez, A. (2016). Adaptación y validación de la scala de bienestar psicológico de Ryff en una muestra de adultos puertorriqueños. Saludy Conducta Humana, 3(1), 1-14.

Rosa-Rodríguez, Y., Negrón, C., Maldonado P., Toledo O., \& Quiñones B. (2015). Dimensiones de bienestar psicológico y apoyo social percibido con relación al sexo y nivel de estudio en universitarios. Avances en Psicología Latinoamericana, 33(1), 31-43.

Ruini, C., Ottolini, F., Rafanelli, C., Ryff, C., \& Fava, G. (2003). La validazione italiana delle Psychological Well-being Scales (PWB). Rivista di psichiatria, 38(3), 117-130.

Ryan, R., \& Deci, E. (2001). On happiness and human potentials: A review of research on hedonic and eudaimonic well-being. Annual Review of Psychology, 52, 141-166. doi:10.1146/annurev.psych.52.1.141

Ryff, C. (1989). Happiness is everything, or is it? Explorations on the meaning of psychological well-being. Journal of Personality and Social Psychology, 57(6), 1069-1081.

Sánchez-Cánovas, J. (2013). Escala de bienestar psicológico. Madrid, España: Tea.

Tomas, J. Meléndez, J., Oliver, A., Navarro, E., \& Zaragoza, G. (2010). Efectos de método en las escalas de Ryff: Un estudio en población de personas mayores. Psicológica, 31(2), 383-400.

Triadó, C., Osuna, M. J., Resano, C. S., \& Villar, F. (2003). Bienestar, adaptación y envejecimiento: Cuando la estabilidad significa cambio. Revista multidisciplinar de gerontología, 13(3), 152-162.

Valenzuela, E. (2015). Bienestar psicológico en una muestra de estudiantes universitarios mexicanos. psicumex, 5(2), 4-19.

Waterman, A. (1993). Two conceptions of happiness: Contrasts of personal expressiveness (eudaimonia) and hedonic enjoyment. Journal of Personality and Social Psychology, 64(4), 678-691. doi:10.1.1.907.5829\&rep=rep1\&type $=$ pdf

Zavala, G., Vidal, G., Castro, S., Quiroga, P., \& Klassen., P. (2006). Funcionamiento social del adulto mayor. Ciencia y Enfermeria, 12(2), 53-62. 